\title{
Editorial \\ Complement and arthritis: another step in understanding
} Michael M Frank and C Garren Hester

Department of Pediatrics, Duke University Medical Center, Durham, NC 27710, USA

Corresponding author: Michael M Frank, frank007@mc.duke.edu

Published: 19 February 2008

Arthritis Research \& Therapy 2008, 10:104 (doi:10.1186/ar2359)

This article is online at http://arthritis-research.com/content/10/1/104

(c) 2008 BioMed Central Ltd

See related research article by Wenderfer et al., http://arthritis-research.com/content/9/5/R114

\begin{abstract}
In a recent research article in Arthritis Research and Therapy ('Analysis of C204 and the C4 binding protein in the MRL/lpr mouse'), Wenderfer and colleagues report that deficiency in C4 binding protein, a down-regulator of the classic pathway of complement, does not affect the development of autoimmune disease. These data support the earlier finding that the alternative pathway, and not the classic pathway, drives disease progression. However, in a milder variant of the MRL/lpr model, the $\mathrm{lpr} / \mathrm{lpr}$ mouse, classic pathway deficiency does contribute toward renal pathology and more severe disease. In this editorial we discuss the factors that may cause such a discrepancy.
\end{abstract}

In a previous issue of Arthritis Research and Therapy, Wenderfer and colleagues [1] analysed the C4 and the C4 binding protein in the MLR/lpr mouse, a well-studied model of immune complex disease with autoimmune tissue damage [2]. The Ipr mutation confers a defect in Fas, with resulting failure of cellular apoptosis, and the MRL defects predispose the mouse to the development of autoimmunity. At an early age these mice develop massive lymphadenopathy, lupus like antibodies, and severe tissue damage.

Organ pathology results, in part, from the formation and organ deposition of immune complexes, the activation of complement, and consequent tissue damage. Previous work has shown that much of this damage is a result of alternative complement pathway (AP) activation [3]. Cross-breeding MRL/lpr mice with complement factor B deficient animals, defective in activation and function of the AP but with the classic complement pathway (CP) intact, leads to amelioration of symptoms, decreased glomerulonephritis, vasculitis, tissue damage and decreased lethality. The CP has not been shown to be a major pathway for complement mediated tissue damage in MRL/lpr mice.

Wenderfer and colleagues [1] have carried this analysis further by examining the effect of $\mathrm{C} 4$ binding protein (C4bp) deficiency in the MRL/lpr model. Their methodology was similar to studies of factor B deficiency. The mutation for C4bp was bred into MRL/lpr mice and these doubly inbred animals were compared to the parent strain MRL/lpr in terms of organ pathology, complement activation, and lethality.

It is known that C4bp is a control protein of the CP and plays little or no role in AP activation [4]. C4bp has a number of actions: it binds primarily to $\mathrm{C} 4 \mathrm{~b}$ to inhibit the continued activation of the CP; it serves to increase the degradation of the CP C3 convertase, consisting of $\mathrm{C} 4 \mathrm{~b}$ and $\mathrm{C} 2 \mathrm{a}$, and thereby decrease the activity of the convertase; it acts as a co-factor for the degradation of $\mathrm{C} 4 \mathrm{~b}$ to $\mathrm{C} 4 \mathrm{~d}$ with complement factor I; and C4bp has been shown more recently to interact with CD40 as well as CD154 in human in vitro models, although the details of these interactions are still not clear $[5,6]$.

In the study by Wenderfer and colleagues [1], the authors report that deficiency in C4bp made little difference to survival, organ pathology, and the extent of autoimmunity in $\mathrm{MRL} / \mathrm{lpr}$ animals. This further supports the central role of the $\mathrm{AP}$ in contrast to the CP. An interesting question relates the findings in this paper and the findings of Prodeus and colleagues [7]. These authors studied the effect of targeting the genes for complement proteins $\mathrm{C} 4$ and $\mathrm{C} 3$ and the complement receptors CD35 and CD21 in the closely related Ipr/lpr model. In the absence of the MRL abnormalities and on the C57/B6 background, these mice develop mild autoimmune disease. They reported that when the gene for C4 or CD21/CD35 was deleted in the lpr/lpr model, but not the gene for C3, there was a marked increase in disease activity and lethality. They reasoned that in this and other disease models, C4 and CD35/CD21 participate in tolerance induction. Presumably, if the gene for C4bp was of great importance in down-regulating the $\mathrm{CP}$, this pathway might be up-regulated upon gene deletion and lead to an increase in

$\mathrm{AP}=$ alternative complement pathway $\mathrm{C} 4 \mathrm{bp}=\mathrm{C} 4$ binding protein $\mathrm{CP}=$ classic complement pathway. 
tolerance induction. However, this was not the case. On the other hand, C4 levels are low in the MRL/lpr model, perhaps preventing $\mathrm{C} 4 \mathrm{bp}$ from regulating $\mathrm{C} 4$ and $\mathrm{CP}$ activity.

The MRL/lpr model may not be the optimal choice for studying the function of $\mathrm{C} 4 \mathrm{bp}$ in mediating tolerance induction. The abnormality is present in mice on an $\mathrm{H}-2^{\mathrm{k}}$ background, which is known to be associated with very low serum C4, as recognized by Wenderfer and colleagues [1]. The MRL/lpr mouse has a defect in Fas mediated apoptosis, one of the multiple checkpoints in the immune response that leads to the deletion of strongly self-reactive lymphocytes [8]. On the MRL background this defect predisposes to severe autoimmunity. The animals have massive enlargement of lymphoid organs. Perhaps the modulating effects contributed by the CP are not evident when massive stimulation of self reactive lymphocytes is induced.

The finding that C4bp deficient MRL/lpr animals have no alteration in autoimmune manifestations of disease confirms the observation that, in this model, the AP plays the dominant role in tissue deposition of immune complexes and organ damage. It is known that in many situations, the AP is activated by the $\mathrm{CP}$, and the $\mathrm{AP}$ provides most of the anaphylotoxins, C3a and C5a, as well as most of the C3 deposited in tissue. In the MRL/lpr model, there may be such high levels of circulating immune complexes in these animals that the AP does not require the CP for optimal activation. More studies should define these issues.

The detailed paper by Wenderfer and colleagues clearly shows that C4bp cannot control the development of autoimmunity in the MRL/lpr model and leads the way to further studies that may help us understand how individual complement pathways contribute to either tolerance or autoimmunity.

\section{Competing interests}

The authors declare that they have no competing interests.

\section{References}

1. Wenderfer SE, Soimo K, Wetsel RA, Braun MC: Analysis of C4 and the $\mathrm{C} 4$ binding protein in the MRL/Ipr mouse. Arthritis Res Ther 2007, 9:R114.

2. Watson ML, Rao JK, Gilkeson GS, Ruiz P, Eicher EM, Pisetsky DS, Matsuzawa A, Rochelle JM, Seldin MF: Genetic analysis of MRL-Ipr mice: relationship of the Fas apoptosis gene to disease manifestations and renal disease-modifying loci. $J$ Exp Med 1992, 176:1645-1656.

3. Watanabe H, Garnier G, Circolo A, Wetsel RA, Ruiz P, Holers VM, Boackle SA, Colten HR, Gilkeson GS: Modulation of renal disease in MRL/lpr mice genetically deficient in the alternative complement pathway factor B. J Immunol 2000, 164:786-794.

4. Blom AM, Villoutreix BO, Dahlback B: Complement inhibitor C4b-binding protein-friend or foe in the innate immune system? Mol Immunol 2004, 40:1333-1346.

5. Brodeur SR, Angelini F, Bacharier LB, Blom AM, Mizoguchi E Fujiwara $H$, Plebani A, Notarangelo LD, Dahlback B, Tsitsikov E, Geha RS: C4b-binding protein (C4BP) activates B cells through the CD40 receptor. Immunity 2003, 18:837-848.

6. Williams KT, Young SP, Negus A, Young LS, Adams DH, Afford $\mathrm{SC}$ : $\mathrm{C} 4 \mathrm{~b}$ binding protein binds to CD154 preventing CD40 mediated cholangiocyte apoptosis: a novel link between complement and epithelial cell survival. PLOS ONE 2007, 2:e159.

7. Prodeus AP, Goerg S, Shen L-M, Pozdnyakova OO, Chu L, Alicot EM, Goodnow CC, Carroll MC: A critical role for complement in maintenance of self-tolerance. Immunity 1998, 9:721-731.

8. Goodnow CC, Cyster JG, Hartley SB, Bell SE, Cooke MP, Healy JI, Akkaraju S, Rathmell JC, Pogue SL, Shokat KP: Self-tolerance checkpoints in B lymphocyte development. Adv Immunol 1995, 59:279-368. 